\title{
A tool for generating testable pop-out effects in geovisual displays
}

\author{
Tumasch Reichenbacher ${ }^{\mathrm{a}, *}$ \\ ${ }^{a}$ Department of Geography, University of Zurich, tumasch.reichenbacher@geo.uzh.ch \\ * Corresponding author
}

Keywords: pop-out, visual attention, geovisual analytics, evaluation

The effectivity and efficiency of geovisual displays is highly dependent on visual search processes to detect, locate, and interpret relevant information for the tasks at hand. To speed up this process in interactive displays, a technique called "pop-out" is usually applied in order to afford visual prompts. By definition, such pop-outs depend on a sufficiently large contrast of the popped-out elements in regard to their contextual elements.

Visual attributes most suitable for attracting attention and hence potentially realising pop-outs are colour, motion, orientation, and size (Wolfe \& Horowitz, 2004). Visual cognition theory suggests that items on a display featuring one of these visual attributes are processed pre-attentively, in parallel, and therefore need less time to be detected.

In previous work we proposed an approach to attention-guiding geovisualisation (Swienty, Reichenbacher, Reppermund, \& Zihl, 2008). Based on visual cognition and design theory we demonstrated how relevant information can be visually encoded, such as to guide the users' attention towards them. Although we collected first empirical evidence for the effectiveness of that approach, further and more detailed investigations are indispensable.

What we propose here is a tool for producing geovisual displays for empirical user studies. Such studies may test popouts, i.e. how contrast changes of display items make them faster detectable, and ultimately increase the effectiveness of such displays. The tool can also randomly select a set of target elements, i.e. those that should be emphasised and pop-out.

For the visual attributes of the display that can be adjusted we focus here on colour, i.e. on its dimensions hue, value, and saturation. In addition, we consider transparency (alpha channel), blur, and motion. Motion is a highly effective attribute for visual attention guiding. To generate a motion effect for elements to be highlighted, a simple onset/offset or blinking may be applied. Although size is a strong pop-up attribute for display elements (as long as their number is not too large and would require too much display estate), we do not take it in consideration here.

Colour affects all parts of a geovisual display. From a design perspective we need to differentiate between the visual background and foreground. Setting colour related parameters can be done for the focal, target relevant elements in the foreground, as well as for the contextual, referential or less focussed elements in the background.

The tool offers setting of colour hue, value, saturation, lightness, and alpha for transparency. In order to adjust the contrast for colour hue and lightness aimed at maximising contrast for pop-out effects, different metrics are calculated. For two different colours ${ }_{\mathrm{c} 1}$ and ${ }_{\mathrm{c} 2}$ :

angular difference of colour hue on the colour wheel: $\Delta \mathrm{h}=\mathrm{h}_{\mathrm{c} 1}-\mathrm{h}_{\mathrm{c} 2}$, where $\mathrm{h}$ is measured in degrees $\left(0^{\circ}-360^{\circ}\right)$

value difference $\Delta \mathrm{v}=\mathrm{v}_{\mathrm{c} 1}-\mathrm{v}_{\mathrm{c} 2}$, where $\mathrm{v}$ is a value $[0,1]$, or $[0,100]$

saturation difference $\Delta \mathrm{s}=\mathrm{s}_{\mathrm{c} 1}-\mathrm{s}_{\mathrm{c} 2}$, where $\mathrm{s}$ is a value $[0,1]$, or $[0,100]$

contrast ratio: $\left(\mathrm{L}_{\mathrm{c} 1}+0.05\right) /\left(\mathrm{L}_{\mathrm{c} 2}+0.05\right)$, where relative colour luminance $\mathrm{L}$ is defined as $\mathrm{L}=0.2126 * \mathrm{R}+0.7152$ $* \mathrm{G}+0.0722 * \mathrm{~B} ; \mathrm{L}_{\mathrm{c} 1}>\mathrm{L}_{\mathrm{c} 2}$

Adjustment of colour hue or value can be applied to individual focal elements, the display background, or regions of the background to generate contrast. Such a regional contrast reduction effect can be applied to clusters of non-focal elements, while keeping the cluster region of highlighted items in focus. Similarly, the contrast difference can be accomplished with lightness, transparency, or blur. 
Currently we are developing a prototype tool based on JavaScript and D3 library (d3js.org). To give you an idea, we consider a typical geovisual display, as depicted in Fig. 1. Let us assume we are interested in the brown dots laid over a choropleth map (Fig. 1).

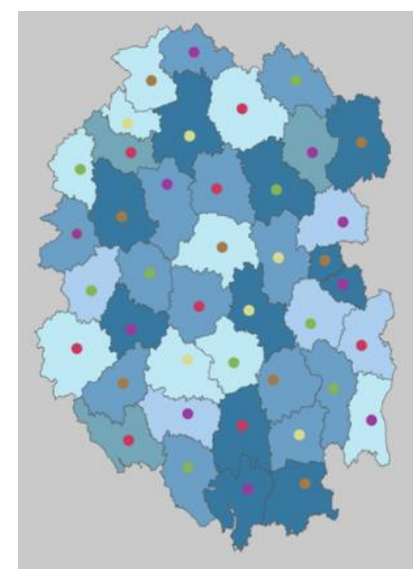

Figure 1. Original geovisual display.

Now, we intensify their colour hue, reduce the contrast of the other dots and likewise the background, i.e. polygon areas of choropleth map (Fig. 2).

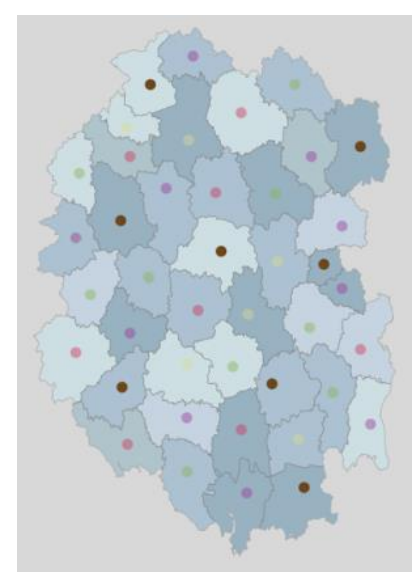

Figure 2. Reducing contrast of background and non-focal elements

In Fig. 3 the brightness of all background polygon colours is maximised, the violet dots are enhanced in their hue contrast and simultaneously all other dots are blurred.

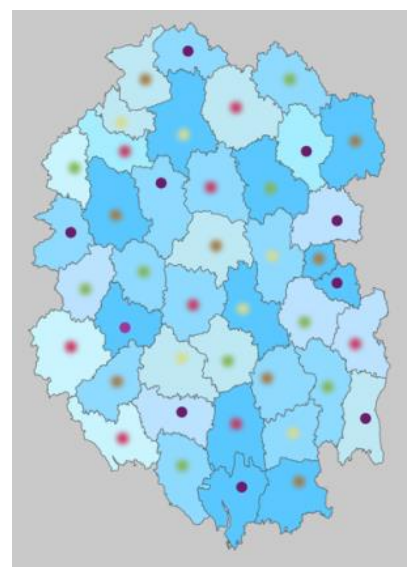

Figure 3. Blurring of non-focal elements.

While the main application of the tool is the simple generation of test displays for empirical evaluation studies it may also find other usages, such as dynamic highlighting in geovisual analytics displays. 


\section{References}

Swienty, O., Reichenbacher, T., Reppermund, S., \& Zihl, J. (2008). The role of relevance and cognition in attentionguiding geovisualisation. The Cartographic Journal, 45(3), 227-238.

Wolfe, J. M., \& Horowitz, T. S. (2004). What attributes guide the deployment of visual attention and how do they do it? Nature Reviews Neuroscience, 5(6), 1-7. 